ISSN 0103-5150

Fisioter. Mov., Curitiba, v. 24, n. 3, p. 455-462, jul./set. 2011 Licenciado sob uma Licença Creative Commons

\title{
Sarcopenia e envelhecimento
}

\author{
Sarcopenia and aging
}

\section{Tatiane da Silva Pícoli ${ }^{[a]}$, Larissa Lomeu de Figueiredo ${ }^{[b]}$, Lislei Jorge Patrizzi ${ }^{[c]}$}

[a] Pós-Graduada em Fisioterapia Cardiorrespiratória pela Universidade de Franca (Unifran), Franca, SP - Brasil, e-mail: tatianepicoli@yahoo.com.br

[b] Especialista em Geriatria pela Universidade Estadual de Campinas (Unicamp), Pós-Graduanda em Fisioterapia Ortopédica, Traumatológica e Desportiva pela Pontifícia Universidade Católica de Minas Gerais (PUC Minas), Poços de Caldas, MG Brasil, e-mail: larissalf.fisio@gmail.com

[c] Professora adjunta do curso de Fisioterapia da Universidade Federal do Triângulo Mineiro (UFTM), Uberaba, MG - Brasil, e-mail: lpatrizzi@uol.com.br

\section{Resumo}

Introdução: 0 envelhecimento está ligado ao grupo de alterações do desenvolvimento que ocorrem nos últimos anos de vida e está associado a alterações profundas na composição corporal. Essa perda relacionada à idade foi denominada "sarcopenia". Objetivos: Avaliar a força muscular no processo de envelhecimento e identificar as variações entre os músculos do abdômen, membros superiores e inferiores. Materiais e métodos: Participaram deste estudo 48 indivíduos, que foram divididos em quatro grupos de acordo com a faixa etária: (G1) 11 a 18 anos, (G2) 20 a 26 anos, (G3) 45 a 60 anos e (G4) 66-82 anos. Os instrumentos de avaliação utilizados foram: 1) esfigmomanômetro (EM) - para análise da força de flexores e extensores da articulação do joelho; 2) flexão de tronco em decúbito dorsal - para avaliação da força dos músculos abdominais (graus 0 a 5); 3 ) dinamômetro Jamar - para avaliação da força de preensão palmar; e 4) dinamômetro Preston Pinch Gauge - para avaliação da força na pinça dos dedos (polegar e indicador). Resultados: Foi observado crescente incremento da força muscular de membros inferiores e superiores com o avançar da idade (G1, G2 e G3) e significativa diminuição da força muscular em todos os segmentos avaliados no G4 quando comparado com o G3. Foi observada importante variação entre a força muscular dos segmentos avaliados e a idade. 0 trabalho sugere que a diminuição da força muscular torna-se 
evidente a partir da sexta década de vida, e que esta apresenta variações entre os músculos do abdômen, membros superiores e inferiores.

Palavras-chave: Envelhecimento. Força muscular. Sarcopenia.

\section{Abstract}

Introduction: The aging process refers to a group of developmental disorders that occur in recent years and is associated with profound changes in body composition. This age-related loss has been termed "sarcopenia". objectives: Evaluate the muscular strength in the ageing process and identify the changes between the abdominal muscles, upper and louver edges. Materials and methods: 48 individuals participated in this study, being divided into four groups according to the age: (G1) 11 to 18 years old, (G2) 20 to 26 years old, (G3) 45 to 60 years old and (G4) 66-82 years old. The evaluation instruments used were: 1) sphygmomanometer (MS) - to analyze the strength of flexors and extensors of the knee joint; 2) trunk flexion in the supine position - to evaluate the strength of the abdominal muscles (grades 0-5); 3) Jamar dynamometer - for evaluation of grip strengthand; 4) Preston Pinch Gauge dynamometer - to evaluate the strength of the gripperfingers (thumb and forefinger). Results: We have observed increasing development of the muscular strength of the upper and louver members with the ageing process (G1, G2 and G3) and significant decrease of the muscular strength of all segments tested in G4 when comparing with G3. We also observed an important variation between the muscular force of the tests segments and the age. The work suggests that the decrease of the muscular force becomes evident from the life sixth decade and that there are variations of strength among the abdominal muscles, upper and louver members.

Keywords: Aging. Muscular strength. Sarcopenia.

\section{Introdução}

A população de idosos está aumentando cada vez mais no Brasil e no mundo, resultando em profundas mudanças na dinâmica demográfica. Nos últimos 60 anos, houve um acréscimo de 15 milhões de indivíduos idosos no País, passando de 4\% para 9\% da população brasileira. Em 2025, estima-se um aumento de mais de 33 milhões, tornando o Brasil o sexto país com maior percentual populacional de idosos no mundo $(1,2)$.

0 envelhecimento está ligado ao grupo de alterações do desenvolvimento que ocorrem nos últimos anos de vida e está associado a alterações profundas na composição corporal. Com a idade, há um aumento na massa de gordura corporal, especialmente com o acúmulo de depósitos de gordura na cavidade abdominal, e uma diminuição da massa corporal magra. Essa diminuição ocorre basicamente como resultado das perdas da massa muscular esquelética. Essa per$\mathrm{da}$, relacionada à idade, foi denominada "sarcopenia" (3). 0 desenvolvimento da sarcopenia é um processo multifatorial que inclui inatividade física, unidade motora remodelada, nivelação de hormônio diminuído e diminuição da síntese de proteína (4).
A sarcopenia estabelece seus sintomas principalmente em indivíduos fisicamente inativos, mas também é vista em sujeitos que permanecem fisicamente ativos ao longo de suas vidas, com isso corroboram fatores pertinentes à saúde pública. Diversos autores verificaram que o treinamento de força pode minimizar ou retardar o processo de sarcopenia para obter significantes respostas neuromusculares (hipertrofia muscular e força muscular), por meio do aumento da capacidade contrátil dos músculos esqueléticos $(4,5)$.

Estudos epidemiológicos $(6,7)$ sugerem que diferentes fatores contribuem para o desenvolvimento da sarcopenia, incluindo alterações hormonais, perda de neurônios motores, nutrição inadequada, inatividade física e baixo grau de inflamação crônica. Essas alterações têm sido apresentadas até mesmo em indivíduos saudáveis, fisicamente ativos, resultando em perda da massa muscular de, aproximadamente, $1 \%$ a $2 \%$ por ano, a partir dos 50 anos de idade (8).

A diminuição da força e da potência do músculo pode influenciar na autonomia, no bem-estar e na qualidade de vida dos idosos (9).

0 objetivo deste estudo foi avaliar a força muscular no processo de envelhecimento e identificar as 
variações entre os músculos do abdômen, membros superiores e inferiores.

\section{Metodologia}

Sujeitos

Quarenta e oito indivíduos participaram voluntariamente deste trabalho, divididos em 4 grupos de 12 participantes, sendo 6 homens e 6 mulheres: (G1) 11 a 18 anos, (G2) 20 a 26 anos, (G3) 45 a 60 e (G4) 66-82 anos. Os participantes foram selecionados nas dependências da Unifran e em residências da cidade de Franca, São Paulo, Brasil. Os critérios de exclusão envolveram pessoas que: praticavam atividade física com frequência, acima de três vezes/semana; tinham artrose severa, déficit cognitivo, obesidade; sofreram acidente vascular cerebral; e faziam uso de órteses para membro inferior ou tinham sofrido fraturas, com ou sem implantes metálicos. Os sujeitos, após serem previamente esclarecidos sobre os propósitos da investigação e procedimentos aos quais seriam submetidos, assinaram um Termo de Consentimento Livre e Esclarecido (Protocolo 088/08).

\section{Instrumentação}

Para a avaliação da força muscular foram utilizados: o esfigmomanômetro BD - para análise da força de flexores e extensores de joelho; o dinamômetro Jamar - para avaliar a integridade funcional dos membros superiores; e o dinamômetro Preston Pinch Gauge - para avaliação de pinça das mãos.

\section{Procedimentos}

a) Esfigmomanômetro (EM): foi utilizado para análise da força de flexores e extensores da articulação do joelho. Antes de realizar o teste, o voluntário recebeu as instruções de como deveria proceder e foi solicitado que realizasse o movimento desejado uma única vez, em cada segmento, como forma de aprendizagem, antes que o EM fosse posicionado e que o teste fosse iniciado. Durante o teste de flexão de joelho, os indivíduos ficaram em decúbito dorsal, com o membro a ser avaliado em uma flexão de $95^{\circ}$ e o outro membro posicionado em extensão. Durante o teste de extensão de joelho, os sujeitos ficaram em decúbito ventral, com os dois membros em extensão, o EM foi posicionado sob o tornozelo, tendo então como base o ponto de apoio (chão) e como topo o tornozelo. 0 aparelho foi insuflado a $140 \mathrm{mmHg}$ como ponto de referência em ambos os testes. 0 voluntário foi induzido a exercer esforço máximo, por meio de estímulos verbais de encorajamento de flexão e extensão do joelho (10).

b) Flexão de tronco na posição supino: solicitouse aos participantes que fizessem a flexão de tronco a partir do decúbito dorsal, com os membros inferiores em extensão, para a avaliação da força dos músculos abdominais. Em todo o teste o paciente ficou com as mãos entrelaçadas atrás da cabeça. 0 paciente realizava o teste até o seu limite. A avaliação seguiu a graduação proposta por Kendall (11), em grau de 0-5.

c) Dinamômetro Jamar: Instrumento confiável e seguro para avaliar a força de preensão palmar. 0 indivíduo foi posicionado sentado com o ombro aduzido em posição neutra, cotovelo fletido a $90^{\circ}$ e antebraço em semipronação. Durante a preensão manual, o braço permaneceu imóvel, havendo somente a flexão das articulações interfalangeana e metacarpofalangeana.

d) Dinamômetro Preston Pinch Gauge: Instrumento para avaliação da força de pinça dos dedos (polegar e indicador). 0 paciente foi posicionado sentado, com o ombro aduzido e em posição neutra e o cotovelo fletido em ângulo reto. 0 polegar e o indicador foram posicionados em discreta flexão da interfalângica proximal, e os demais dedos não envolvidos na pinça foram mantidos em semiflexão.

Para todos os testes foram solicitados aos participantes três repetições sucessivas, sendo o resultado final a média desses valores. Este estudo foi aprovado pelo Comitê de Ética em Pesquisa da Universidade de Franca (Protocolo 088/08).

\section{Análise estatística}

Foi realizada uma análise descritiva das variáveis estudadas. A normalidade da distribuição da amostra foi testada aceitando-se valores de $p>0,05$. Caso 
indicativo de normalidade na distribuição, o teste ANOVA foi utilizado para comparação dos dados normais e para os dados normais após transformação (teste de Johnson).

Para os dados que não indicaram normalidade na distribuição da amostra mesmo após transformação, foi utilizado o teste de Kruskal Wallis (não paramétrico), sendo estabelecido o nível de significância de $\mathrm{p}<0,05$ para todas as análises estatísticas. Já para a análise das variações encontradas entre os músculos do abdômen, membros superiores e inferiores foram comparados os valores máximos obtidos (G3 e G4 FAb) com o G4 (Tabela 2).

\section{Resultados}

Foi observado crescente incremento da força muscular de membros inferiores e superiores com o avançar da idade (G1, G2 e G3) e significativa diminuição da força muscular em todos os segmentos avaliados no G4 quando comparado com o G3. Observou-se importante variação entre a força muscular dos segmentos avaliados e a idade (Tabela 2).
Foi observada diferença significativa ( $5 \%$ de significância), em pelo menos um grupo, para as variáveis FMId $(p=0,02), \operatorname{PPd}(p=0,01), \operatorname{Pd}(p=0,04)$, Pe $(p=0,01)$, FAb $(p=0,001)$, sendo consideradas estatisticamente iguais às variáveis FMIe $(p=0,09)$, EMIe $(p=0,2)$, EMId $(p=0,06)$, PPe $(p=0,2)$ entre os grupos avaliados (Tabela 1 ).

\section{Discussão}

Estudos evidenciam que a força muscular atinge seu pico por volta dos 30 anos de idade e é satisfatoriamente preservada até os 50 anos (12). Durante a senescência, ocorre diminuição da força muscular, em uma taxa que varia de $20 \%$ a $40 \%$, na população entre 70-80 anos. Ao considerar idosos nonagenários, essa taxa é agravada e a redução da força é maior do que $50 \%$ (13). Contudo, um declínio da força ocorre entre os 50 e 60 anos de idade e evolui lentamente, com um grau bem mais rápido de diminuição após os 60 anos (14).

Sowers et al. (15), a partir de um estudo observacional, apresentaram uma relação direta entre a

Tabela 1 - Médias obtidas entre os grupos nas variáveis estudadas

\begin{tabular}{lccccccccc}
\hline & $\begin{array}{c}\text { FMld } \\
(\mathbf{m m H g})\end{array}$ & $\begin{array}{c}\text { FMle } \\
(\mathbf{m m H g})\end{array}$ & $\begin{array}{c}\text { EMId } \\
(\mathbf{m m H g})\end{array}$ & $\begin{array}{c}\text { EMle } \\
(\mathbf{m m H g})\end{array}$ & $\begin{array}{c}\text { PPd } \\
(\mathbf{K g})\end{array}$ & $\begin{array}{c}\text { PPe } \\
(\mathbf{K g})\end{array}$ & $\begin{array}{c}\text { Pd } \\
(\mathbf{K g})\end{array}$ & $\begin{array}{c}\text { Pe } \\
(\mathrm{Kg})\end{array}$ & $\begin{array}{c}\text { FAb } \\
\left(\mathbf{0}-5^{\star}\right)\end{array}$ \\
\hline G1 (11-18 anos) & 210,8 & 201,8 & 186,3 & 182,5 & 33,8 & 35,1 & 5,7 & 5,7 & 4,2 \\
G2 (20-26 anos) & 221,8 & 225 & 206,1 & 198 & 42,6 & 40 & 7,5 & 6,5 & 4,5 \\
G3 (45-60 anos) & 237,4 & 232,6 & 214,2 & 203,5 & 45,6 & 43,8 & 7,8 & 7,2 & 3,8 \\
G4 (66-82 anos) & 192 & 199,5 & 199 & 198,1 & 34,4 & 33,1 & 4,7 & 4,0 & 2,2 \\
\hline
\end{tabular}

Legenda: FMId = flexão membro inferior direito; FMle = flexão membro inferior esquerdo; EMld = extensão membro inferior direito; EMle = extensão membro inferior esquerdo; PPd = preensão palmar direita; $\mathrm{PPe}=$ preensão palmar esquerda; $\mathrm{Pd}=$ pinça direita; $\mathrm{Pe}=$ pinça esquerda; $F A b=$ força abdômen; * $=$ Kendall.

Fonte: Dados da pesquisa.

Tabela 2 - Dados referentes à perda (\%) de força muscular no G4 nos segmentos estudados

\begin{tabular}{lccccccccc}
\hline & $\begin{array}{c}\text { FMld } \\
(\mathbf{m m H g})\end{array}$ & $\begin{array}{c}\text { FMle } \\
(\mathbf{m m H g})\end{array}$ & $\begin{array}{c}\text { EMld } \\
(\mathbf{m m H g})\end{array}$ & $\begin{array}{c}\text { EMle } \\
(\mathbf{m m H g})\end{array}$ & $\begin{array}{c}\text { PPd } \\
(\mathbf{K g})\end{array}$ & $\begin{array}{c}\mathrm{PPe} \\
(\mathrm{Kg})\end{array}$ & $\begin{array}{c}\mathrm{Pd} \\
(\mathbf{K g})\end{array}$ & $\begin{array}{c}\text { Pe } \\
(\mathbf{K g})\end{array}$ & $\begin{array}{c}\mathrm{FAb} \\
\left(\mathbf{0}-5^{\star}\right)\end{array}$ \\
\hline G4 (66-82 ano) & $19,13 \%$ & $14,24 \%$ & $7,1 \%$ & $2,7 \%$ & $24,6 \%$ & $24,5 \%$ & $39,8 \%$ & $44,5 \%$ & $51,2 \%$ \\
\hline
\end{tabular}

Legenda: FMId = flexão membro inferior direito; FMle = flexão membro inferior esquerdo; EMld = extensão membro inferior direito; EMle = extensão membro inferior esquerdo; $\mathrm{PPd}=$ preensão palmar direita; $\mathrm{PPe}=$ preensão palmar esquerda, $\mathrm{Pd}=$ pinça direita; $\mathrm{Pe}=$ pinça esquerda; FAb = força abdômen; * $=$ Kendall).

Fonte: Dados da pesquisa. 
perda de massa muscular comum ao envelhecimento, diminuição de força de membros inferiores e velocidade da marcha, além do aumento do tempo da fase de apoio duplo na deambulação. Segundo esse estudo, a perda de massa magra, apesar de estar relacionada ao desempenho funcional, parece estar mais fortemente ligada à força de membros inferiores (15). No entanto, no presente estudo foi observado o crescente incremento da força muscular de membros inferiores e superiores com o avançar da idade (G1, G2 e G3) e significativa diminuição da força muscular em todos os segmentos avaliados no G4 quando comparado com o G3. Observou-se importante variação entre a força muscular dos segmentos avaliados e a idade (Tabela 2).

Deschenes (12) e Kauffman (14) citam que, após a quinta década de vida, a taxa de progressão de redução da força se dá em torno de $8 \%$ a $15 \%$ por década, e tanto homens quanto mulheres exibem o mesmo padrão de diminuição da força durante o envelhecimento. Contudo, investigações longitudinais têm revelado diminuição da força em idosos maior do que as apresentadas por estudos transversais.

Homens e mulheres idosos com menor atividade física têm também menor massa muscular e maior prevalência de incapacidade física (16). A prática regular de exercícios desde a juventude lentifica a perda muscular do idoso. E a intervenção mais eficaz para a prevenção e recuperação da perda muscular são os exercícios de resistência (17). A habilidade para desempenhar atividades da vida diária, a massa e a força muscular são bastante relacionadas entre si. No estudo do Novo México, mulheres e homens sarcopênicos tinham, respectivamente, 3,6 e 4,1 maiores chances de incapacidade quando comparados àqueles com maior massa muscular. Da mesma forma, o uso de órteses e a maior frequência de quedas foram mais relatados pelos idosos com menor massa muscular. Recentemente, o mesmo grupo descreveu maior prevalência de sarcopenia em mulheres idosas com grande prejuízo funcional.

O estudo ainda mostrou a importância de identificar indivíduos obesos e sarcopênicos, uma vez que o aumento de gordura corporal e a estabilidade do peso dificilmente suscitam interesse sobre a perda muscular. Nessa população, tanto obesidade quanto sarcopenia se associaram a limitações funcionais, incapacidade e maior número de quedas, particularmente no sexo feminino. A população sarcopênica e obesa (S-O) tinha alto risco para três ou mais incapacidades físicas quando comparada aos somente sarcopênicos (S) ou somente obesos (0). Entre os homens, o risco foi de 8,7 (S-0) versus 3,8 (S) versus 1,3 (0). Nas mulheres, foi de 12 (S-0) versus 3 ( $\mathrm{S}$ ) versus 2,2 (0) (18).

Os comprometimentos adicionais na função muscular, associados às doenças agudas ou crônicas, às hospitalizações por trauma ou por cirurgia e à inatividade, podem acelerar o declínio da força muscular (19).

Com o aumento da população idosa e da expectativa de vida, começam a surgir com maior frequência doenças e comorbidades relacionadas ao processo de envelhecimento, como, por exemplo, a sarcopenia (14). 0 termo não deve ser considerado em casos de perda muscular associados a processos inflamatórios, perda de peso ou doenças em estágios avançados (20).

A sarcopenia do envelhecimento está associada ao declínio progressivo da massa e, consequentemente, da função muscular (força, potência e resistência). Esse déficit pode ser relacionado a uma contração muscular inadequada, seja em função de alterações nas proteínas actina e miosina ou por um estresse oxidativo nas células. 0 início e a progressão dessa perda muscular estão relacionados à perda de miócitos via apoptose, essa perda é mais pronunciada nas fibras do tipo II $(21,22)$.

Segundo Rebelatto et al. (23), as alterações anatomofisiológicas características do processo de envelhecimento são muito importantes do ponto de vista funcional do sistema muscular. A sarcopenia ocorre principalmente pela diminuição do peso muscular e diminuição da área de secção transversal. Consequentemente, o idoso terá menor qualidade em sua contração muscular, menor força, menor coordenação dos movimentos e, provavelmente, maior probabilidade de sofrer acidente (por exemplo, quedas).

Segundo Deschenes (7), o decréscimo no número de fibras musculares é a principal causa de sarcopenia, embora a atrofia da fibra, particularmente do tipo II, também esteja envolvida.

Para Rebelatto et al. (19), com o passar dos anos as fibras de contração rápida ou do tipo II vão diminuindo em número e volume e as fibras de contração lenta ou do tipo I também diminuem, mas em menor proporção que as primeiras. Esse fato talvez explique a menor velocidade que se observa nos movimentos do idoso.

Sabe-se que a força gerada por um músculo não é diretamente proporcional à quantidade de fibra 
muscular presente nele. Com base nesse fato, recentemente o termo dinapenia $($ dyna $=$ força; penia $=$ perda) foi proposto por Clark e Manini (24) para definir a perda específica da força muscular relacionada ao envelhecimento, e para dissociar a perda de força da perda de massa muscular. Essa recente informação, indicando a dissociação entre massa muscular e a força, suporta a noção de que outras adaptações de função fisiológica (celular, neural e metabólica) são mediadoras da perda de força relacionada à idade. Isso sugere que ganhos de força, a curto prazo, não estão relacionados a fatores associados à capacidade intrínseca do músculo. Esses fatores constituem uma interação complexa entre mudanças nas propriedades de ativação das unidades motoras, bem como a adaptação no comando central para o aprendizado (24).

Em 2002, Janssen, Heymsfield e Ross (25) desenvolveram um novo método para mensuração da sarcopenia relativa utilizando uma estratégia para correção da massa gorda, de modo a não influenciar no resultado do cálculo. 0 índice de músculo esquelético (IME) foi definido com a fórmula: massa muscular esquelética (estimada por meio da análise de bioimpedância) dividida pela massa corporal $\times$ 100, o que irá representar a porcentagem de massa muscular sobre a massa corporal do indivíduo. Nesse mesmo estudo foi elaborado um sistema de classificação da sarcopenia, a fim de graduar a perda de massa muscular, bem como o impacto dessa perda nas atividades funcionais de indivíduos sarcopênicos. A sarcopenia classe I é considerada presente em indivíduos em que o IME se encontra entre um e dois desvios-padrões dos valores de adultos jovens, e a sarcopenia classe II está presente em indivíduos que possuem IME abaixo de dois desvios-padrões dos valores de adultos jovens. Essa forma de classificação é semelhante à forma utilizada para se classificar a densidade mineral óssea em normal, osteopenia e osteoporose. Clinicamente, a classificação da sarcopenia elaborada por Janssen et al. (25) parece não ser muito utilizada, porém tem sido fortemente adotada por pesquisadores.

Outro estudo demonstrou que a força do joelho aliada ao equilíbrio é preditora do desenvolvimento de limitações severas na caminhada. Essa limitação é definida como velocidade de marcha menor que 0.4 $\mathrm{m} / \mathrm{s}$, inabilidade para caminhar um quarto de milha ou inabilidade total de caminhar. Assim, podese concluir que a lentidão da velocidade de marcha pode identificar idosos com diminuição da força e potência muscular de membros inferiores, com declínio funcional e risco para limitações de membros inferiores (26).

Um instrumento bastante utilizado para se estimar a força muscular global em idosos é a força de preensão palmar. De forma geral, os idosos que apresentam força de preensão manual reduzida são sedentários, possuem déficits de massa corporal, apresentam problemas de saúde e limitações funcionais em atividades que exigem a participação dos membros superiores e inferiores. Portanto, essa medida deixa de ser apenas uma simples medida da força da mão, limitada à avaliação do membro superior, e passa a apresentar outras implicações clínicas (27).

Os valores da força de preensão manual têm mostrado associação significativa com a incapacidade funcional: indivíduos com menores valores de força apresentaram menor velocidade de andar e risco duas vezes maior de incapacidade de autocuidado, sugerindo que a medida dessa variável na idade adulta pode servir como fator prognóstico de risco de incapacidade física na velhice (28).

A incapacidade funcional exerce grande efeito negativo no bem-estar individual, gerando mais necessidade de assistência à saúde e cuidados por longos períodos. Incapacidade funcional é comumente definida como a restrição da capacidade do indivíduo de desempenhar atividades normais da vida diária. Refere-se também a limitações específicas no desempenho de papéis socialmente definidos e de tarefas dentro de um ambiente sociocultural e físico particular. Estão incluídas as atividades básicas e instrumentais de vida diária, os papéis no trabalho, nas atividades não ocupacionais, nos recreativos ou de lazer (29).

Para Hunter et al. (30), o decréscimo da função muscular, com a consequente diminuição da funcionalidade, pode tornar-se um ciclo vicioso, visto que a diminuição da função muscular induz a uma baixa no nível de atividade física, que, por sua vez, causa um decréscimo ainda maior na função muscular com consequências diretas na qualidade de vida do idoso.

À medida que a população envelhece torna-se cada vez mais evidente a necessidade do estudo dos fatores associados à sarcopenia, visto que melhores e mais eficazes estratégias e intervenções de prevenção e tratamento poderão ser desenvolvidas para minimizar a incapacidade e otimizar a independência de idosos (19). 


\section{Conclusão}

O trabalho sugere que a diminuição da força muscular torna-se evidente a partir da sexta década de vida, e que tal diminuição apresenta variações entre os músculos do abdômen, membros superiores e inferiores.

\section{Referências}

1. Parahyba MI, Simões CCS. A prevalência de incapacidade funcional em idosos no Brasil. Ciênc Saúde Coletiva. 2006;11(4):967-74.

2. Freitas EV. Tratado de geriatria e gerontologia. 2a ed. Rio de Janeiro: Guanabara Koogan; 2006.

3. Guccione AA. Fisioterapia geriátrica. 2a ed. Rio de Janeiro: Guanabara; 2000.

4. Roth SM, Ferell RF, Hurley BF. Strength training for the prevention and treatment of sarcopenia. J Nutr Health Aging. 2000;4(3):143-55.

5. Hurley BF, Roth SM. Strength training in the elderly: effects on risk factors for age-related diseases. Sports Med. 2000;30(4):249-68.

6. Dreyer HC, Volpi E. Role of protein and amino acids in the pathophysiology and treatment of sarcopenia. J Am Coll Nutr. 2005;24(2):140S-45S.

7. Vandervoort AA. Aging of the human neuromuscular system. Muscle Nerve. 2002;25(1):17-25.

8. Hughes VA, Frontera WR, Roubenoff R, Evans WJ, Singh MAF. Longitudinal changes in body composition in older men and women: role of body weight change and physical activity. Am J Clin Nutr. 2002;76(2):473-81.

9. Assumpção CO, Souza TMF, Urtado CB. Treinamento resistido frente ao envelhecimento: uma alternativa viável e eficaz. Anuário Prod Acad Docente. 2008; 2(3):451-76.

10. Delgado C, Fernandes JF, Barbosa FP, Oliveira HB. Utilização do esfigmomanômetro na avaliação da força dos músculos extensores e flexores da articulação do joelho em militares. Revista Brasileira Méd Esporte. 2004;10(5):362-6.

11. Kendall PF, McCreary KE, Provance GP, Rodgers IM, Romane AW. Músculos: provas e funções. 5a ed. Barueri: Manole; 2007. p. 170.
12. Deschenes MR. Effects of aging on muscle fibre type and size. Sports Medicine. 2004;34(12):809-24.

13. Garcia PA. Sarcopenia, mobilidade funcional e nível de atividade física em idosos ativos da comunidade. [dissertação]. Belo Horizonte: Escola de Educação Física, Fisioterapia e Terapia Ocupacional. Universidade Federal de Minas Gerais; 2008.

14. Kauffman TL. Manual de reabilitação geriátrica. Rio de Janeiro: Guanabara Koogan; 2001.

15. Sowers MR, Crutchfield M, Richards K, Wilkin MK, Furniss A, Jannausch M, et al. Sarcopenia is related to physical functioning and leg strength in middle-aged women. J Gerontol A Biol Sci Med Sci. 2005;60(4):486-490.

16. Vans WJ. Effects of exercise on senescent muscle. Clin Orthop Relat Res. 2002;(403 Suppl):S211-20.

17. Doherty TJ. Invited review: aging and sarcopenia. J Appl Physiol. 2003;95(4):1717-27.

18. Baumgartner RN. Body composition in healthy aging. Ann N Y Acad Sci. 2000;904:437-48.

19. Silva TAA, Frisoli A Jr., Pinheiro MM, Szejnfeld VL. Sarcopenia associada ao envelhecimento: aspectos etiológicos e opções terapêuticas. Rev Bras Reumatol. 2006;46(6):391-7.

20. Roubenoff R. Origins and clinical relevance of sarcopenia. Can J Appl Physiol. 2001;26(1):78-89.

21. Zhong S, Chen CN, Thompson LV. Sarcopenia of ageing: functional, structural and biochemical alterations. Rev Bras Fisioter. 2007;11(2):91-7.

22. Dreyer HC, Volpi E. Role of protein and amino acids in the pathophysiology and treatment of sarcopenia. J Am Coll Nutr. 2005;24(2):140S-45S.

23. Rebelatto JR, Morelli JGS. Fisioterapia geriátrica: a prática da assistência ao idoso. São Paulo: Barueri; 2004.

24. Clark BC, Manini TM. Sarcopenia =/= dynapenia. J Gerontol A Biol Sci Med Sci. 2008;63(8):829-834.

25. Janssen I, Heymsfield SB, Ross R. Low relative skeletal muscle mass (sarcopenia) in older persons is associated with functional impairment and physical disability. J Am Geriatr Soc. 2002;(5):889-96.

26. Rantanen T, Guralnik JM, Ferrucci L, Penninx BW, Leveille S, Sipilä S, et al. Coimpairments as predictors of severe walking disability in older women. J Am Geriatr Soc. 2001;49(1):21-7. 
27. Shechtman O, Mann WC, Justiss MD, Tomita M. Grip strength in the frail elderly. Am J Phys Med Rehabil. 2004;83(11):819-26.

28. Rantanen T, Penninx B, Masaki K, Lintunen T, Foley D, Guralnik JM. Depressed mood and body mass index as predictors of muscle strength decline in old men. J Am Geriatr Soc. 2000;48(6):613-7.

29. Rabelo DF, Cardoso CM. Auto-eficácia, doenças crônicas e incapacidade funcional na velhice. PsicoUSF. 2007;12(1):75-81.
30. Hunter GR, McCarthy JP, Bamman MM. Effects of resistance training on older adults. Sports Med. 2004; 34(5):329-48.

Recebido: 29/09/2010

Received: 09/29/2010

Aprovado: 13/05/2011

Approved: 05/13/2011 